\title{
Sortir du lot : Quelle expression anaphorique est utilisée lors de l'extraction d'un référent de son groupe référentiel ?
}

\author{
Estèle Dupuy \\ Université de Poitiers, CESCM, CNRS-UMR 6223 \\ estele.dupuy@univ-poitiers.fr
}

\section{Préambule}

Il est aujourd'hui montré que la période du Moyen Français (désormais MF) présente un grand intérêt quant à une étude de la continuité référentielle. D'une part, parce qu'à cette période se développent ou apparaissent certains procédés de référenciation inexistants auparavant comme la cataphore ${ }^{1}$, d'autre part parce que certaines ambiguités morphologiques - il/ils/ilz - offrent une approche d'autant plus intéressante quant aux stratégies mises en place afin de pallier d'éventuels faux-sens ou ambiguïtés sémantiques - sachant qu'en MF la désinence verbale n'est pas ambiguë.

Ainsi, cette étude diachronique a été réalisée à partir d'un corpus de trois textes - les Quinze joies de mariage (QJM) de la fin du $14^{\text {ème }}$ siècle, les Chroniques de Froissart (Chro.) des environs de 1400 et les Mémoires de Commynes (Mém.) de la fin du $15^{\text {ème }}$ siècle $^{2}$. Ce corpus couvre une période d'un siècle et permet une comparaison tri-dimensionnelle : une comparaison de genres littéraires, diachronique, voire dialectale dans une moindre mesure ${ }^{3}$. La comparaison de genre repose sur une opposition entre les $Q J M$ et les deux autres textes. Les $Q J M$ correspondent à une succession de récits brefs dont les protagonistes sont des personnages génériques représentés sous la forme de syntagmes nominaux résomptifs (c'est-àdire de valeur sémantique non particularisante mais généralisante valant pour la catégorie sous-jacente) et non mentionnés par leur nom propre, l'objectif étant de «ne pas nommer les personnages » (Lagorgette, 2002 : 507-526). La particularité essentielle de ce texte pour notre étude sur le récit est que les chaines anaphoriques que nous y rencontrons présentent des référents peu nombreux (six seulement) qui, en situation de co-présence, offrent rarement les mêmes caractéristiques référentielles, limitant ainsi les risques de concurrence référentielle. De fait, le maillage référentiel de ce texte est beaucoup plus simple que celui des deux autres textes qui correspondent au genre du récit historique. Dans ceux-ci, les protagonistes sont des personnages historiques (réels ou fictifs) présentés par leur nom propre par souci de vérité historique, d'où un effet de clarté référentielle. Cependant, le maillage référentiel de ces deux textes est beaucoup plus complexe puisque le nombre de référents y est beaucoup plus élevé ( 25 dans les Chro. et 46 dans les Mém.) et ces référents potentiellement co-présents tour à tour sont pour une grande majorité de mêmes caractéristiques référentielles (de genre masculin) ce qui augmente fortement les risques de concurrence référentielle en cas de reprise pronominale. Ainsi la comparaison entre les $Q J M$ et les Chro. permet de dégager les écarts propres au genre et à la complexité référentielle du texte et celle entre les Chro. et les Mém. conduit à observer les écarts propres à une évolution diachronique.

L'étude que nous proposons ici s'inscrit dans une recherche plus large ${ }^{4}$ sur la continuité référentielle en MF qui a montré que le choix des expressions anaphoriques qui y contribuent, ne relève pas d'un choix subjectif mais répond à des régularités de fonctionnement précises que nous pouvons qualifier de règles. Ces règles syntactico-sémantiques forment une combinatoire 5 permettant d'expliciter l'utilisation des expressions anaphoriques formant la chaîne anaphorique envisagée par nous comme la combinaison de l'anaphore et de la coréférence réunissant linéairement toutes les chaînes de références (Schnedecker, 1997) ayant une même entité comme référent et permettant le maintien de la continuité référentielle en s'appuyant sur le choix des expressions anaphoriques ${ }^{6}$, sur la sémantique verbale et la syntaxe propositionnelle. L'une de ces cinq règles est la règle de concurrence référentielle qui montre que lorsque plusieurs référents co-présents sont mentionnés en contexte, la concurrence référentielle entre eux 
n'existe que s'ils ont les mêmes caractéristiques référentielles (genre, nombre, nature sémantique ${ }^{7}$ et même personne verbale, non ambiguë en MF). Lorsque ces référents co-présents diffèrent par l'une de leurs caractéristiques référentielles, cela se traduit par l'utilisation de l'anaphore pronominale ou «zéro »; la sémantique verbale selon l'approche de Cl. Blanche-Benveniste (1984) contribue à la réalisation du calcul inférentiel. En revanche, lorsque les référents co-présents ont des caractéristiques référentielles identiques, l'anaphore nominale apparaît à chaque reprise après entrelacs de chaînes anaphoriques. Cette règle est plus fréquemment vérifiée dans des contextes où le maillage référentiel est simple, soit, lorsque le nombre de référents co-présents ayant de mêmes caractéristiques référentielles est faible (deux à trois). Lorsque le maillage référentiel est plus complexe (trois référents co-présents ou plus), les autres règles de la combinatoire, prenant en compte la répartition des référents sur les rôles valentiels, prennent souvent le pas sur la précédente règle ${ }^{8}$, sans l'évincer.

Les questions qui nous intéressent ici découlent de la précédente thématique et de l'observation des relations inter-référentielles, notamment des occurrences de référents extraits d'un groupe référentiel. Lorsqu'un référent est inclus dans un groupe référentiel, il entre souvent dans une catégorie. A l'inverse, un référent peut aussi être séparé du groupe référentiel dont il était un des constituants : il vaut alors pour lui-même sur le plan référentiel. Puisqu'aucune ambiguïté n'a été relevée, comment est alors présenté par son expression anaphorique ce référent extrait pour que la continuité référentielle soit préservée à la fois pour le groupe référentiel dont le référent est extrait mais aussi pour le référent extrait ? Comment part-on d'un groupe référentiel pour arriver à un référent extrait valant pour lui-même sans "perdre » en route l'un des deux, le groupe référentiel ou le référent extrait? Comment se fait le lien référentiel entre le référent extrait et le groupe référentiel dont il est extrait? Quels indices peut donner l'expression anaphorique de ce référent extrait, sur lui-même, sur la constitution du groupe référentiel, sur l'application de la règle de concurrence référentielle ? Il s'agit donc d'observer quelle expression anaphorique est utilisée lors de son extraction et dans quelle mesure le choix de l'expression anaphorique obéit ou n'obéit pas à la règle de concurrence référentielle.

\section{Introduction}

Nos observations portent sur l'extraction d'un référent ou d'un sous-groupe référentiel du groupe référentiel plus large, dit matrice, dans lequel il est inclus?.

\begin{tabular}{|c|c|c|c|}
\hline & QJM $^{10}$ & Chro. & Mém. \\
\hline $\begin{array}{c}\text { Nbre minimum de } \\
\text { gpes réf }\end{array}$ & 10 & 54 & 52 \\
\hline
\end{tabular}

Tableau 1 : Nombre d'occurrences présentant un groupe référentiel suivi d'une extraction ${ }^{11}$

Les référents sont beaucoup plus nombreux chez Commynes qu'ils ne le sont chez Froissart ou dans les $Q J M^{12}$. Il serait impossible de comptabiliser tous les groupes référentiels mis en place dans les Chro.: une approximation fiable serait de l'ordre de 54 groupes référentiels au minimum mais nous ne retiendrons que les 25 occurrences qui donnent lieu à l'extraction d'un référent ou à la scission du groupe référentiel matrice. Pour les Mém., nous n'avons fait le même choix: ne sont retenues que les 46 occurrences de groupes référentiels qui subissent une scission ou l'extraction de l'un de leurs référents (sur les 52 occurrences de groupes référentiels) ${ }^{13}$.

L'analyse que nous allons exposer montre que l'expression anaphorique utilisée lors de l'extraction d'un référent ou de la scission d'un groupe référentiel est fonction de la nature des entités constituant le groupe référentiel et de leur degré de définitude (allant du groupe nominal mentionné par un SN indéfini ou défini classifiant à une énumération de noms propres). Nous présenterons les groupes référentiels en allant du moins défini au plus défini. Nos résultats dépendront de la constitution du groupe référentiel. 


\section{Configurations constitutionnelles du groupe référentiel à l'extraction d'un référent}

Ces configurations sont au nombre de quatre et sont répertoriées dans le tableau ci-dessous :

\begin{tabular}{|c|c|c|c|}
\hline $\begin{array}{l}\text { Entités } \\
\text { constitutives } \\
\text { du groupe } \\
\text { référentiel }\end{array}$ & & & $\begin{array}{l}\text { Groupe référentiel } \\
\text { masculin mentionné par: }\end{array}$ \\
\hline \multirow[t]{6}{*}{ - identifiées } & \multicolumn{2}{|c|}{$\begin{array}{c}\text { Configuration } \mathbf{A} \text { : } \\
\text { Groupe référentiel }=\text { plusieurs entités référentielles } \\
\text { non identifiées et non définies }\end{array}$} & Un SN indéfini \\
\hline & \multirow{2}{*}{\multicolumn{2}{|c|}{$\begin{array}{c}\text { Configurations B : } \\
\text { Groupe référentiel = entités référentielles non } \\
\text { identifiées et non définies }+ \text { une entité référentielle } \\
\text { définie }\end{array}$}} & $\begin{array}{l}\text { Un nom classifiant } \\
\text { catégorisant }\end{array}$ \\
\hline & & & $\begin{array}{l}\text { Un SN coordonné à un } \\
\text { nom ou un pronom }\end{array}$ \\
\hline & \multirow{3}{*}{$\begin{array}{l}\text { Configuration C: } \\
\text { Groupe d'entités } \\
\text { référentielles } \\
\text { identifiées et définies } \\
\quad+\text { une entité } \\
\text { référentielle définie }\end{array}$} & $\begin{array}{l}\text { Différence de genre : } \\
\text { "Référent individuel } \\
\text { masculin + Référent } \\
\text { individuel féminin » }\end{array}$ & $\begin{array}{ll}- & \mathrm{SN}+\mathrm{SN} \\
- & \text { Pronom personnel } \\
\text { de P6 } \\
\text { - } & \text { Anaphore zéro } \\
\text { avec le verbe de } \\
\text { P6 }\end{array}$ \\
\hline & & $\begin{array}{l}\text { Différence de nombre : } \\
\text { « référent individuel + groupe } \\
\text { référentiel » }\end{array}$ & $\mathrm{SN}+\mathrm{SN}$ \\
\hline & & $\begin{array}{l}\text { Mêmes caractéristiques } \\
\text { référentielles : « référent } \\
\text { individuel + référent } \\
\text { individuel + référent } \\
\text { individuel... » }\end{array}$ & Un SN catégorisant \\
\hline + identifiées & \multicolumn{2}{|c|}{$\begin{array}{l}\text { Configuration D : } \\
\text { Référent unique identifié dont est extraite une } \\
\text { composante interne }\end{array}$} & $\begin{array}{l}\text { Un nom (propre ou } \\
\text { commun) }\end{array}$ \\
\hline
\end{tabular}

Tableau 2 : Configurations constitutionnelles du groupe référentiel à l'extraction d'un référent

\subsection{Configuration $A$ : entités référentielles non identifiées et non définies}

Un groupe référentiel exprimé uniquement sous la forme d'une catégorie (sans entité référentielle identifiée et définie) subira une scission qui présentera les référents qui le composent, sous une forme indéfinie (Kleiber, 1994 : 23 sq.).

Ainsi, dans l'énoncé (1) tiré des $Q J M^{14}$, le groupe référentiel mentionné sous la forme nominale les gallans représente une catégorie référentielle dont les entités constitutives ne sont pas préalablement présentées de manière individuelle et définie.

(1) Lors les gallans, qui la voient bien abillee et bien emparlee, se avencent chacun endroit soy de luy ouffrir raison l'un plus que l'autre quar jolis maintien et gaillart de femme donne hardement a couart. L'un lui presente beaux moz plaisans et gracieux, l'autre lui marche dessus le pié ou lui estraint la main, l'autre la regarde d'un regart trenchant et piteux de cousté, l'autre lui presente ung ennel, ung dyamant ou ung rubi, par lesquelles choses la dame peut assez savoir de leurs voulentés, si elle est telle que elle entende raison aucunement. (QJM, Seconde joye, p. 15-16, 1. 49 à $74^{15}$ ) 
Lors de l'éclatement du groupe référentiel, les entités qui le constituaient répercutent cette indéfinitude à travers leur expression anaphorique : au moyen de pronoms indéfinis : chacun (singulier collectif à michemin entre le groupe référentiel et sa scission), L'un, l'autre... en énumération. L'utilisation de ces pronoms réalise parfaitement la continuité référentielle entre les entités référentielles indéfinies et le groupe référentiel catégorisant dont elles sont issues.

On retrouve ce choix d'expressions anaphoriques dans nos autres textes. Ainsi dans les Mém. et les Chro. ${ }^{16}$, certains groupes référentiels sont présentés sous une forme nominale évoquant une caractéristique commune sans qu'il soit possible d'identifier les entités référentielles qui composent le groupe référentiel. Pour exemple, dans (2),

(2) Toutesfoiz, avec ce petit de gens qu'il [= duc de Bourgogne] peult amasser, il tira a Dorlens avecques quatre ou cinq cens chevaulx seullement, en intencion de garder Amyens de tourner; et la fut cinq ou six jours que ceulx d'Amyens marchandoient, car l'armee du Roy estoit auprés, qui se presenta devant la ville. Et ung coup la refuserent, car une partie de la ville tenoit pour ledict duc, lequel y envoya faire son logis. Et s'il eust eu gens pour y oser entrer en personne, il ne l'eust jamais perdue, mais il n'y ousoit entrer mal acompaigné, combien qu'il en fust requis de plusieurs de la ville. Quant ceulx qui estoient contre luy veirent sa dissimulation, et qu'il n'estoit fort, executerent leur entreprinse et mysdrent ceulx du Roy dedans. (Mém., livre III, chap. II, p. 222, 1. 2-15)

le groupe référentiel désigné sous une forme nominale, ceulx d'Amyens, dont les entités constitutives non-identifiées individuellement - sont catégorisées par une de leurs caractéristiques définitionnelles, 'appartenir à la ville d'Amyens', stipulée par le complément de détermination d'Amyens est ensuite repris par anaphore « zéro » à travers la personne 6 du verbe refuserent avant d'être subdivisé par extraction, en sous-groupes référentiels à partir du groupe matrice (Schéma de (2)) :

Schéma de (2)

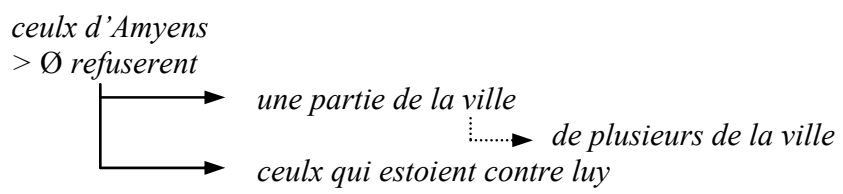

Le premier sous-groupe référentiel $\mathrm{G}_{\mathrm{p}} 1$, une partie de la ville, est mentionné par anaphore nominale mais ne permet pas l'identification individuelle des entités référentielles qui le composent : il fonctionne aussi par catégorisation.

Le second sous-groupe référentiel $\mathrm{G}_{\mathrm{p}} 1$, de plusieurs de la ville, est extrait du sous-groupe référentiel $\mathrm{G}_{\mathrm{p}} 1$, une partie de la ville. Il fonctionne par catégorisation mais affiche un pluriel marqué par le pronom indéfini quantifiant plusieurs qui amorce une individualisation des référents constitutifs du groupe référentiel sans pour autant permettre d'accéder à leur identité référentielle individuelle.

Le troisième sous-groupe référentiel $\mathrm{G}_{\mathrm{p}} 2$, ceulx qui estoient contre luy, fonctionne aussi par catégorisation et est extrait du groupe référentiel matrice. Il est complémentaire du premier sous-groupe $\mathrm{G}_{\mathrm{p}} 1$. 
Les sous-groupes référentiels $\mathrm{G}_{\mathrm{p}} 1$ et $\mathrm{G}_{\mathrm{p}} 2$ sont constitués d'entités référentielles qui ne peuvent être mises en commun d'un groupe à l'autre; cependant, rien dans le contexte ne permet d'affirmer que leur addition constitue la totalité du groupe matrice $\mathrm{G}_{\mathrm{p}} 0$, ceulx d'Amyens.

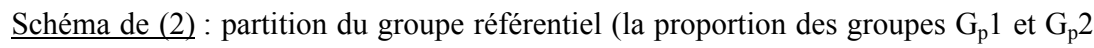
étant indéfinissable)

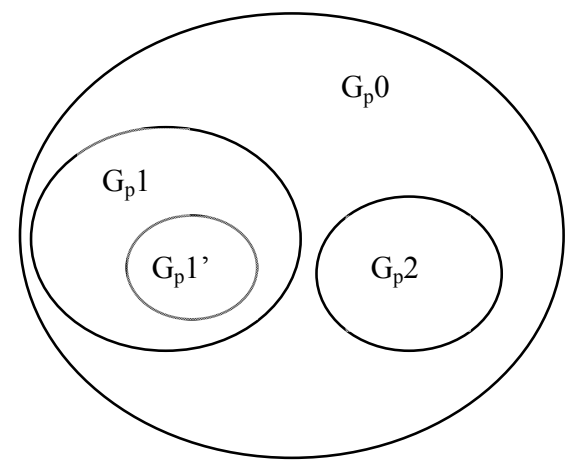

D'autres groupes référentiels - mentionnés par anaphore nominale et dont les entités référentielles sont non-identifiables individuellement - peuvent être scindés en sous-groupes complémentaires dont l'addition équivaut à la totalité du groupe référentiel matrice tout en conservant, dans leur expression anaphorique, l'indéfinitude des sous-groupes référentiels extraits. Il en va ainsi dans (3), où le groupe référentiel l'armee du duc de Bourgongne est présenté sous une forme nominale qui ne permet pas d'identifier précisément les entités référentielles qui le composent. Ces entités référentielles sont uniquement catégorisées par leur appartenance au groupe référentiel armee du duc de Bourgongne.

(3) Dieu voulut ainsi dispouser des choses que ceste nuyt sourdit une grande tormente, et telle qu'il faillut que l'armee du duc de Bourgongne fouyst; et coururent les ungs des navyres en Escosse, les aultres en Hollende ; (Mém., livre III, chap. V, p. 238, 1. $1-4)$

Ce groupe référentiel $\mathrm{G}_{\mathrm{p}} 0$ est ensuite scindé en deux sous-groupes référentiels qui portent également les marques de cette indéfinitude dans leur expression anaphorique : $\mathrm{G}_{\mathrm{p}} 1$, les ungs des navyres et $\mathrm{G}_{\mathrm{p}} 2$, les aultres. Ces deux sous-groupes sont complémentaires et leur addition constitue la totalité du groupe référentiel matrice $\mathrm{G}_{\mathrm{p}} 0$.

Schéma de (3): Indiquée par des pointillés et la ligne courbe, la frontière indéfinie entre ces deux groupes référentiels. Cette indéfinitude est sans conséquence sur la continuité référentielle ${ }^{17}$.



Dans les Mém. ${ }^{18}$ et les Chro., nous rencontrons également des groupes référentiels dont les entités sont non identifiées mais quantifiées et qui apparaissent sous forme nominale indéfinie catégorisante, comme en (4) :

(4) Et fist son amas de gens d'armes en la chité de Nantes et la environ, et furent bien ..XVII douse mille honmes de piet parmi les arbalestriers. Entre ces ..CCCC.. chevaliers avoit ..XXIII...baneres.( Chro., livre I, chap. CCXLVII, p. 812, 1. 65-72) 


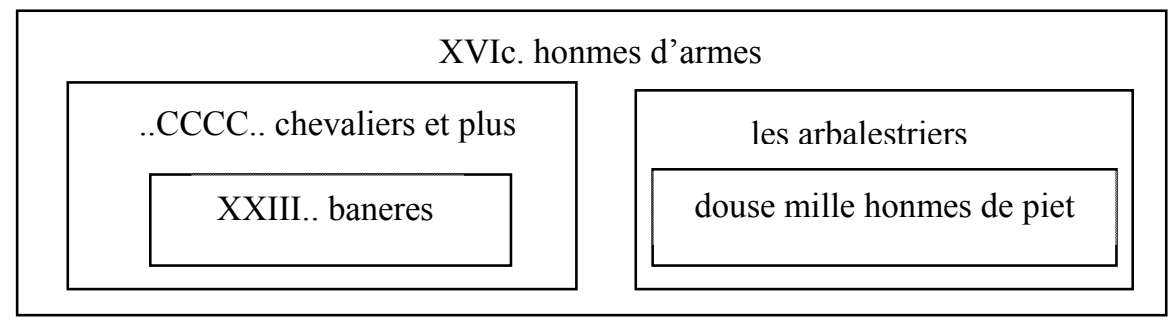

L'extraction d'un sous-groupe référentiel et la scission d'un groupe référentiel matrice en sous-groupes référentiels dont les entités sont indéfinies et présentées uniquement par catégorisation ne perturbent pas la continuité référentielle car ces opérations sont étayées par l'utilisation d'une forme nominale ou pronominale (pronom indéfini prolongeant l'indéfinitude). De plus, les indications chiffrées ne font que délimiter plus précisément les frontières de chaque groupe référentiel sans pour autant donner d'indication sur l'identité individuelle des référents qui les constituent.

\subsection{Configuration $B$ : entités référentielles non identifiées et non définies + une entité référentielle définie mentionnée sous forme nominale}

Cette constitution de groupe référentiel est la plus fréquente, principalement dans les Mém. Le groupe référentiel peut apparaître sous la forme de :

\subsubsection{Groupe référentiel mentionné par un nom commun classifiant}

Dans les $Q J M$, nous ne rencontrons que ce type d'occurrence. Il apparaît également dans les $C h r o .{ }^{19}$ mais n'est pas représenté dans les Mém.

Un groupe référentiel exprimé uniquement sous la forme d'une catégorie (sans entité référentielle identifiée et définie) peut inclure un référent défini et identifié mais non exprimé explicitement dans le groupe matrice. Mais dans ce cas, le référent inclus étant le seul référentiellement identifiable et défini du groupe, son extraction ne nécessite pas l'utilisation d'une anaphore nominale puisque aucun des référents implicites du groupe référentiel ne peut entrer en concurrence référentielle avec lui. Pourtant, une anaphore nominale peut être utilisée. Ainsi, pour (5), un référent est extrait du groupe référentiel touz ses servans et servantes sur le mode du connu par anaphore nominale, la chamberiere qui la garde, souscatégorie incluse dans l'hyperonyme servantes.

(5) II [= le mari] entre a l'oustel et trouve touz ses servans et servantes instruiz a la poste de la dame, car aultrement ilz n'y demoureroient point, tant fussent ilz bons et loyaulx, et demande comment elle le fait, et la chamberiere qui la garde lui respond que elle est trop malalde et que oncques puis qu'il partit que elle ne mengea, mais elle est ung pou apaisee devers le soir, combien que tout est mensonge. (QJM, Tierce joye p. 20-22, 1.95 à 159$)$

La complémentation du référent par la relative qui la garde ajoute une précision référentielle et sémantique sachant qu'il peut y avoir plusieurs chambrières au sein de la maison. Pour cette occurrence nous entrons dans le cadre de l'anaphore associative (Kleiber, 2001), le groupe référentiel touz ses servans et servantes recouvre plusieurs fonctions des personnels de la maison dont celle de 'chambrière' et l'utilisation d'un déterminant défini présentant le référent comme connu suggère que le groupe référentiel matrice induit la présence d'une chambrière chargée principalement de la maîtresse de maison. 


\subsubsection{Groupe référentiel mentionné par un SN incluant une coordination}

Cette construction est absente des QJM. En revanche, elle s'observe dans les Chro. et les Mém. Pour ces occurrences, le groupe référentiel est composé d'un sous-groupe référentiel catégorisant indéfini (sans entité référentielle identifiée et définie) auquel s'ajoute un référent défini et identifié exprimé explicitement dans le groupe matrice.

\section{$\underline{\mathrm{SN}}=\mathrm{Nom}+\mathrm{GN}$ coordonné}

Dans les Mém., les groupes référentiels présentent le plus souvent un référent identifié par un nom propre et coordonné à un groupe référentiel dont les entités référentielles ne sont pas individuellement identifiées. Ainsi dans (6), le groupe référentiel est constitué par coordination du référent défini son lieutenant [...] Mons ${ }^{r}$ de Waucloe au groupe référentiel plusieurs de ses serviteurs domesticques dont les entités ne sont pas individuellement identifiées mais catégorisées en tant que serviteurs domesticques du conte de Warvic. A l'extraction du référent défini, celui-ci apparait sous la forme nominale ledict seigneur de Waucloe. Or cette forme nominale peut être due à la co-présence d'un référent de mêmes caractéristiques référentielles, 'le conte de Warvic' qui peut entrer en concurrence référentielle avec le référent 'Mons ${ }^{\mathrm{r}}$ de Vaucloe ${ }^{20}$ :

(6) Et dedans estoit son lieutenant [= le seigneur de Vaucloe] en la ville, appellé Mons ${ }^{\mathrm{r}}$ de Waucloe et plusieurs de ses serviteurs domesticques, qui, en lieu de le [= le conte de Warvic] recueillir, luy tirerent de grands coups de canon. Et, estants a l'ancre la devant, acoucha la duchesse de Clarence, fille dudict conte de Warvic, d'un filz. A grand peyne voulut consentir ledict seigneur de Waucloe que on luy portast deux flaccons de vin. (Mém., livre III, chap. IV, p. 233, 1. 5-12)

\section{$\underline{\mathrm{SN}}=$ pronom $+\mathrm{GN}$ coordonné}

Cette construction apparaît surtout dans les Chro. Nous y rencontrons à quatre reprises ${ }^{21}$ des groupes référentiels composés d'un référent identifié masculin singulier animé humain présenté en groupe référentiel par anaphore pronominale et coordonné à un sous-groupe référentiel apparaissant sous forme nominale comme ils et ses consauls de l'occurrence (7). A chaque fois, le pronom personnel qui est utilisé est de personne 3 : il apparaît sous la forme, ils, dont le $-s$ est la marque du cas sujet singulier, le pronom étant alors assimilé au substantif auquel il est coordonné ${ }^{22}$. Notons également que les quatre occurrences présentent leur groupe référentiel en fonction de sujet grammatical. Pour exemple, dans (7), le groupe référentiel est constitué d'un référent défini et identifié, li rois de France, repris par anaphore pronominale avec le pronom personnel de personne 3, ils, auquel est coordonné un $\mathrm{SN}$, et ses consauls. A l'extraction du référent 'roi de France' du groupe référentiel, celui-ci apparaît sous forme nominale, li rois de France.

(7) Qant li rois de France entendi ces paroles et les responses des $\underline{\underline{\text { Flamens }}}$, si les laissa ester, et comsidera assés lor maniere et vei bien que $\underline{\underline{\text { ils }}}$ n'en averoit aultre cose et que point n'enteroit en euls sus cel estat pour ratraire a sa volenté, fors par le moiien dou duch de Braibant; mais pour le present, ils et ses consauls estoient cargiet de si grant cose que a ceste des $\underline{\underline{\text { Flamens }}}$, il ne pooient entendre. Si mist li rois de France ceste cose en sousfrance tant que a une aultre fois, et entendi a voloir lever le siege de Calais. (Chro., livre I, chap. CCXLVIII, p. 823, 1. 104-113)

Cependant, nous ne pouvons pas généraliser ce phénomène puisqu'il ne se vérifie pas pour les trois autres occurrences dont le groupe référentiel apparaît selon ce modèle :

- pour deux d'entre elles, ils et ses gens (Chro., p. 824, 1. 9) et ils et li siis bourgeois (Chro., p. 845, 1. 102-103), l'extraction du référent fait appel à un pronom personnel de personne 3, il.

- Pour la dernière, ils ne ses gens (Chro., p. 833, 1. 48), il n'y a pas extraction.

Un pronom ou un nom entrant en coordination détachée avec un GN

Cette construction apparaît dans les Mém. uniquement. Elle semble être une version plus souple de la formation du groupe référentiel par coordination. Dans (8), l'adjonction du référent 'ce roy Edouard' au 
groupe référentiel quelzques sept ou huyt cens personnes est renforcé par le complément circonstanciel d'accompagnement avecques luy - luy ayant pour référent 'le roi Edouard'. Ce groupe référentiel poursuit sa chaîne anaphorique par anaphore pronominale avec quililz (sujet de alloient) et par anaphore «zéro » avec les verbes avoient. L'extraction du référent 'roi Edouard' se fait par anaphore nominale ce pouvre roy (elle peut être liée à son entrée dans un intermède narratif «Bien estoit estrange ») :

(8) Ainsi fouyt ce roy Edouard [...] et quelzques sept ou huyt cens personnes avecques luy, qui n'avoient aultre habillement [...]. Et si n'avoient ne croix ne pille, ne n'y sçavoient a grand peyne ou ilz alloient. Bien estoit estrange a ce pouvre roy (car ainsi [se] pouvoit il bien appeller) de ainsi s'en fouyr et estre persecuté de ses propres serviteurs. (Mém., livre III, p. 239, 1. 32 - p. 240, 1. 2)

La présentation du référent identifié sous forme pronominale lors de son adjonction à un groupe référentiel reste exceptionnelle dans les Mém. et ne se fait jamais avec un pronom personnel sujet ${ }^{23}$.

\subsection{Configuration $C$ : entités référentielles identifiées et définies + une entité référentielle définie}

Les occurrences de cette catégorie présentent des expressions anaphoriques qui se laissent expliquer par la règle de concurrence référentielle: dès lors que le groupe référentiel est constitué d'entités référentielles ne présentant pas les mêmes caractéristiques référentielles, l'extraction de l'une d'entre elles peut se faire par anaphore pronominale ou «zéro » sans ambiguïté. Si dans les $Q J M$, toutes les occurrences de groupe référentiel constitué d'entités référentielles identifiées présentent la fusion d'entités différant par leur genre, dans les Chro. deux occurrences présentent des référents différant par leur nombre (un référent ajouté à un groupe référentiel) mais aussi des groupes référentiels constitués d'entités de même genre, nombre et nature comme c'est également et exclusivement le cas des occurrences relevées dans les Mém.

\subsection{1 « Référent individuel masculin + référent individuel féminin » identifiés : différence de genre}

Les référents présentant une différence de genre peuvent être fusionnés en un groupe référentiel « référent individuel + référent individuel» Ce groupe référentiel peut être mentionné sous la forme (six occurrences dans les $\left.Q J M^{24}\right)$ :

- d'un SN résomptif,

- d'un pronom personnel de P6

- ou d'une anaphore « zéro » avec la P6 du verbe

La fusion des deux référents peut se faire sous forme nominale comme dans (9) - où le groupe référentiel est constitué de deux référents préalablement définis et identifiés qui sont coordonnés sous la forme d'un $\mathrm{SN}$ : le jeunes homs et sa femme -, à l'aide d'un pronom personnel sujet ilz comme dans (10) ou par anaphore zéro comme dans (11).

(9) La tierce joye de mariage si est que, après ce que le jeunes homs et sa femme, qui est jeune, ont bien prins de plaisances et delectacions, elle devient grousse, (QJM, Tierce joye p. 18, 1. 1 à 4)

(10) Et s'il n'y avoit que cela, si fault il qu'il aille avant, car elle [= feme de plus grant lignage qu'il n'est ou plus jeune (1. 5-6)] ne vieult pas abesser sa lignee, et le mary se tient moult honnouré de ce que Dieu lui fist la grace qu'il la peut avoir.Et avient souvent que, quant ilz se courrocent ensemble, elle lui dit par maniere de menaces que ses amis ne la la lui baillerent pas pour la paillarder (QJM, Quinte joye, p. 33, 1. 15 à 27)

(11) Et va la dame a l'eiglise et le gallant y est [...] II se approuche d'elle et parlent ensemble, mes elle ne lui vieult rien accorder et ne vieult rien prendre de lui, mais 
tourjours elle lui respond en telle maniere qu'il cognoist bien que elle le ame bien et que elle ne craint que deshonneur, dont il est bien aise. (QJM, Quinte joye, p. 44-45, 1. 293 à 408)

Dans (9), (10) et (11), l'extraction d'un des deux référents se fait par anaphore pronominale sans concurrence référentielle. De plus, le pronom servant à l'extraction présente des caractéristiques morphologiques orientant le calcul inférentiel.

Néanmoins, l'anaphore pronominale n'est pas obligatoire, d'autres facteurs comme le changement de point de vue avec mise en parallèle de deux situations (coordination) comme dans (12) ou le franchissement d'une étape narrative marqué par Après lesquelx plaisirs dans (13) engendrent l'emploi d'une forme nominale (Schnedecker, 1997).

(12) Mais s'il avient que ceste dame vieult avoir robe ou aultre chouse de son mary, et scet bien ses condicions, [...] Et quant $\underline{\underline{\mathbf{l} z}}$ sont en leur chambre en leur granz deliz et plaisances et que la dame voit qu'il a affere d'elle, elle lui fait si bonne chiere et si estrange que c'est merveilles, [...] veult. (QJM, Quinte joye, p. 38-39, 1. 198 à 208)

(13) Quant la dame a amy a sa plaisance et ilz se pouent trouver ensemble, et est a tart, ilz se font tant de joies que nul ne le pourroit dire, tant que le fait du mary n'est rien prisé. Après lesquelx plaisirs la dame prent autant de plesir en l'esbat de sonmary [...] (QJM, Quinte joye, p. 35-36, 1. 97 à 102)

\subsection{2 "Référent individuel + groupe référentiel (dont les entités sont identifiées) » : différence de nombre}

Des référents identifiés présentant une différence de nombre peuvent être fusionnés en un groupe référentiel du type « référent individuel + groupe référentiel (dont les référents sont identifiés) ». Ce groupe référentiel sera mentionné sous la forme de deux SN coordonnés.

On trouve ce type d'occurrence uniquement dans les Chro. Les référents différant par leur nombre, la règle de concurrence référentielle continue de s'appliquer à l'extraction de l'un d'eux.

Deux occurrences ${ }^{25}$ des Chro. concernent un groupe référentiel auquel on réfère par un SN comprenant un nom propre et un GN coordonné faisant référence à un sous-groupe référentiel (dont les entités ont été identifiées dans le contexte de gauche), comme dans (14) avec le groupe référentiel messires Gautiers et li ..VI.. bourgois.

(14) Si passerent oultre messires Gautiers et li ..VI.. bourgois, et s'en vint devant le roi et li dist en langage englois: (Chro., livre I, chap. CCLV, p. 846, 1. 148-150)

L'extraction du référent identifié, messires Gautiers, se fait par anaphore zéro à travers la personne 3 des verbes vint et dist, ce, du fait que le groupe référentiel messires Gautiers et li ..VI.. bourgois inclut le référent messire Gautiers par coordination tout en le plaçant à l'écart du sous-groupe li .. VI.. bourgois. Ainsi, la différence de nombre entre le référent messire Gautiers et le sous-groupe référentiel li ..VI.. bourgois, évite la concurrence référentielle entre ces référents en cas de scission du groupe référentiel matrice.

\subsection{3 « Référent individuel + Référent individuel + Référent individuel... » identifiés et catégorisés : mêmes caractéristiques référentielles}

Certains groupes référentiels sont présentés sous la forme d'un SN catégorisant incluant deux ou plusieurs référents identifiés individuellement par un nom propre au préalable.

Cette configuration est présente dans les Chro. et les Mém. Dans (15), les référents constitutifs du groupe ont les mêmes caractéristiques référentielles : 


\begin{abstract}
assamblé, furent parvenu a deus lieues priés de l'oost des François, il se logierent au lonch de celle riviere [...]. Qant il orent soupé assés legierement, il comsidererent lor

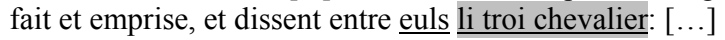

Chils consauls fu tenus, et ordonné tout chil qui se departiroient et chil qui demorroient. Et s'armerent et monterent as chevaus ou conduit des trois chevaliers desus nonmés et cevaucierent tout quoiement; et droit a l'eure de mienuit, il se bouterent en l'oost de messire Carle de Blois a l'un des costés, et i fissent de premieres venues grant damage, et ocirent, mehagnierent et abatirent biaucop de gens. Li hoos se conmença a estourmir, et se courirent armer tout chil qui le plus apparilliet estoient, et a venir a force sus ces Englois et Bretons: liquel se quidierent partir, qant il veirent l'oost toute estourmie et retraire arriere, mais il ne porent; [...], et ne porent porter ne soustenir tant que pour celle heure, le fait des François. Et i fu pris et moult dolereusement navrés mesires Thomas d'Angourne, et se sauva, au mieuls que il pot, $\underline{\text { messires Jehans de Hartecelle, }}$ et aussi fist messirires_tangis__dou__hastelle et se departirent de la bataille; mais il i laissierent une partie de lors gens mors et pris. (Chro., livre I, chap. CCXLVII, p. 813-814, 1. 103-141)
\end{abstract}

Dans $(15)^{26}$, l'extraction individuelle des trois référents messires Thomas d'Angourne, messires Jehans de Hartecelle et messires Tangis dou Chastiel désolidarisés du groupe référentiel 'ces Englois et Bretons' se fait par anaphore nominale pour chacun d'eux : mesires Thomas d'Angourne, pour le premier ; puis, pour le second, par «ana-cataphore $»^{27}$ avec le pronom personnel P3, il, immédiatement suivi d'une reprise nominale visant à éviter toute ambiguité référentielle, messires Jehans de Hartecelle, et enfin par anaphore nominale pour le troisième référent, messires Tangis dou Chastel.

Il en va de même dans les Mém. où les référents peuvent être coordonnés et présentés en groupe référentiel :

- sous forme de noms propres comme dans (16) où la concurrence référentielle impose la reprise nominale, le Roy, du référent extrait du groupe :

(16) Et afin que on congnoisse les habilités de quoy on use en France, vueil compter comment cecy fut guidé, car le Roy et ledict duc y furent deceuz tous deux, et en recommença la guerre, qui dura bien treize ou quatorze ans, qui despuis fut bien dure et bien aspre. Il est vray que le Roy desiroit fort que ces villes feissent nouvelleté ; et print ses couleurs, [...] villes, (Mém., livre III, chap. I, p. 217, 1. 21 - p. 218, 1. 3)

- sous une forme nominale catégorisante dont les référents ont été identifiés au préalable comme dans (17) où l'anaphore pronominale, tous deux, fusionne les référents le Roy et ledict duc [de Bourgogne $]^{28}$ en un groupe référentiel dont la relation interne de commutativité est renforcée par l'anaphore pronominale suivante, l'un pour l'autre. A nouveau, l'extraction de l'une des deux entités constitutives du groupe référentiel se fait par anaphore nominale, ledict duc de Bourgongne, afin d'éviter la concurrence référentielle :

(17) [le connestable Saint Pol répond à la demande de secours du duc de Bourgogne en lui disant que si ce dernier veut son aide contre le Roi, il doit donner sa fille en mariage au duc de Guyenne (cf. Mém., livre III, p. 222-223)]

Car combien que le Roy fust lors son maistre, si avoit il la pluspart de son son vaillant et ses enfans soubz ledict duct mais tousjours a usé de ces termes de les [= duc de Bourgogne et le Roi Louis XI] vouloir tenir en craincte tous deux et l'un pour l'autre ; dont mal luy est prins. [...]

Aprés que ledict duc de Bourgongne eut ouy responce du connestable, congneut bien que en luy ne trouveroit nulle amytié (Mém., livre III, chap. II, p. 224, 1. 2-14)

\title{
3.4 Configuration $D$ : Extraction d'une composante interne d'un référent unique et identifié
}

Ce type d'extraction a été précédemment abordé lors d'une étude des synecdoques ${ }^{29}$. Nous ne l'avons relevé que chez Commynes. Pour exemple, dans (18), 


\begin{abstract}
(18) il [= le duc de Bourgogne] taschoit a tant de choses grandes qu'il n'avoit point le temps a vivre pour les mectre a fin, et estoient choses presque impossibles, car la moictié d'Europe ne l'eust sceu contenter. II avoit assés hardiment pour entreprandre toutes choses ; sa personne povoit asséz porter le travail qui luy estoit necessaire ; il estoit assés puissant de gens et d'argent, mais il n'avoit point assés de sens ny de malice pour conduyre ses entreprises. (Mém., livre III, chap. III, p. 229, 1. 29-36)
\end{abstract}

le référent 'duc de Bourgogne' est une entité unique identifiée qui poursuit sa chaîne anaphorique par anaphore pronominale et zéro. Or, cette entité référentielle en théorie indivisible se voit partitionnée. Le référent 'sa personne' (au sens de 'ses qualités physiques') mentionné sous forme nominale est extrait du référent 'duc de Bourgogne' et désigne une partie intrinsèque de ce référent. L'anaphore nominale $s a$ personne est requise du fait de l'introduction de ce référent en première mention (Kleiber, 2001 : 226228). Cette extraction ne perturbe pas la chaîne anaphorique du référent 'duc de Bourgogne' qui se poursuit par anaphore pronominale. De plus, cette sous-partie référentielle est présentée en interdéfinition avec le référent dont elle est extraite par le biais du déterminant possessif $s a$. En outre, il a été montré $^{30}$ que ce type de référent ne donne jamais suite à une chaîne anaphorique puisqu'il ne peut constituer qu'une partie de référent et non un référent à part entière.

\title{
4 Conclusion
}

Le choix de l'expression anaphorique utilisée lors de l'extraction d'un référent d'un groupe référentiel dépend de la nature des entités qui constituent le groupe référentiel et du degré d'identification de ces entités.

Si le groupe référentiel est constitué d'entités non identifiables, alors l'extraction de l'une d'elles ou d'un sous-groupe référentiel se fera au moyen d'un pronom ou d'un SN indéfini qui continuera de marquer cette indéfinitude.

Ensuite, la règle de concurrence référentielle prend le relais :

- Si le groupe référentiel est constitué d'entités référentielles identifiées associées entre elles ou à un sous-groupe référentiel, la différence de genre ( $Q J M)$, de nombre (Chro. et Mém.) ou de nature (Mém. avec le phénomène de synecdoque) entre les référents formant le groupe référentiel, élimine la possibilité de concurrence référentielle : l'anaphore pronominale ou zéro sont donc utilisées lors de l'extraction de l'un des référents constitutifs du groupe référentiel.

- Si le groupe référentiel est constitué d'entités référentielles identifiées individuellement (coordonnées ou rassemblées par catégorisation sous la forme d'un SN) et qu'elles présentent les mêmes caractéristiques référentielles, il y a alors concurrence référentielle entre ces référents et leur extraction du groupe référentiel se fera au moyen d'une forme nominale (souvent un nom propre ou un $\mathrm{SN}$ résomptif).

L'extraction d'un référent fonctionne donc selon les critères de la règle de concurrence référentielle. Il faut noter une certaine souplesse dans l'application de ces constantes dans les Chro. puisque nous trouvons des occurrences qui présentent une forme nominale là où la règle de concurrence référentielle autoriserait une forme pronominale. En revanche, ces constantes sont plus régulièrement suivies dans les Mém. Il semble donc que diachroniquement, ces tendances et l'application de la règle de concurrence référentielle se renforcent afin de préserver au mieux la continuité référentielle.

\section{Bibliographie}

Ariel, M. (1990). Accessing Noun Phrases Antecedents. London : Routledge.

Blanche-Benveniste, C. (1990). Grammaire première et grammaire seconde : l'exemple de en. Recherches sur le français parlé, 10, 51-73. 
Blanche-Benveniste, Cl., Deulofeu, J., et alii (1984). Pronoms et syntaxe : l'approche pronominale et son application au français. Paris : S.E.L.A.F.

Charolles, M. (1988). Les plans d'organisation textuelle : périodes, chaînes, portées et séquences. Pratiques, 57, 3-13.

Charolles, M. (1990). L'anaphore associative. Problèmes de délimitation. Verbum, XIII, 3, 119-148.

Charolles, M. (2002). La référence et les expressions référentielles en français. Paris : Ophrys, 228-237.

Chastain, C. (1975). Reference and contexte, Language, Mind and Knowledge. Minneapolis : University of Minnesota Press.

Combettes, B., (2003). Grammaticalisation de la phrase complexe et évolution de la cataphore. Langage et référence. Uppsala : Mélange à K. Jonasson, Acta Universitatis Upsaliensis, 105-114.

Corblin, F. (1985). Anaphore et interprétation des segments nominaux. Thèse d'Etat. Université de Paris VII.

Corblin, F. (1995). Les formes de reprise dans le discours, Anaphores et chaînes de référence. Rennes: Presses Universitaires.

Cornish, F. (2000). L'accessibilité cognitive des référents, le centrage d'attention et la structuration du discours : une vue d'ensemble. Verbum, XXII, 1, 7-30.

De Mulder, W. et Schnedecker, C. (2001). Les référents évolutifs entre linguistique et philosophie : Actes des Journées d'étude des 11 et 12 septembre 1997. Paris : Klincksieck.

Dupuy-Parant, E. (2006). La continuité référentielle en moyen français : règles syntactico-sémantiques. Thèse de Doctorat. Le Mans : Université du Maine.

Dupuy-Parant, E. (2008a). Le verbe au cœur de la continuité référentielle : Unité structurante et maillage inter-verbal des systèmes valentico-référentiels. In : Fagard, B. Prévost, S. Combettes B. et Bertrand O. (éds) (2008). Evolutions en français, études de linguistique diachronique. Paris : Peter Lang, vol. 86, 63-85.

Dupuy-Parant, E. (2008b). La portée de la comparaison, de la métaphore et de la métonymie sur la continuité référentielle en moyen français (section «diachronie, histoire de la langue »), Actes en ligne du ler Congrès mondial de linguistique française (CMLF-08). www.linguistiquefrançaise.org.

Dupuy-Parant, E. (2008c). Règles syntactico-sémantiques de la continuité référentielle : fréquence d'application à la traduction de Premierfait du Decameron de Bocasse, Le moyen français 63. 43-72.

Dupuy-Parant, E. (à paraître a). L'émergence de la cataphore dans la prose du moyen français, Actes de la $18^{\text {ème }}$ Conférence Internationale de Linguistique Historique. Montréal : John Benjamins Publisching Company.

Dupuy, E. (2010). Les constructions référentielles inter-définitionnelles en Moyen Français : les emplois du déterminant possessif et/ou du complément de détermination. In Combettes, C. Guillot, E. Oppermann-Marsaux, S. Prévost \& A. Rodríguez Somolinos (éds), Le changement en français. Etudes de linguistique diachronique. Bern : Peter lang. 143-162.

Givón, T. (1983). Topic Continuity in Discourse : an Introduction. Topic Continuity in Discourse. A Quantitative Cross-Language Study. Amsterdam : Benjamins Publishing Company, 1-41.

Grosz, B., Weinstein, S. et Joshi, A. K., (1995). Centering : a framework for modeling the local coherence of discourse. Computational Linguistics, 21, 2, 203-225.

Gundel, J.-K., Hedberg, N. et Zacharski, R. (1993). Cognitive status and the form of referring expressions. Language, 69, 2, 274-307.

Kesik, M. (1989). La cataphore. Paris : PUF.

Kleiber, G., Schnedecker, C. et Tyvaert, J.-E. (eds.) (1997). La continuité référentielle. Paris : Klincksieck.

Kleiber, G. (1994). Pronom et anaphore : il dépend-il de son antécédent ? Travaux linguistiques, 27, 167-182.

Lagorgette, D. (2002). Représentations du sens linguistique. Allemagne : Muenchen.

Maillard, M. (1987). Comment ça fonctionne. Thèse d'Etat, Université de Paris X-Nanterre.

Maillard, M. (1974). Essai de typologie des substituts diaphoriques, Langue française, 21, 55-71. 
Marchello-Nizia, Ch. (1997). La Langue française aux XIV $V^{\grave{e}}$ et $X V^{\grave{e}}$ siècles. Paris : Nathan.

Riegel, M. (1994). Article défini, anaphore intraphrastique et relations partie-tout. In : Schnedecker, C., Charolles, M., Kleiber G. et David J. (eds), L'anaphore associative, aspects linguistiques, psycholinguistiques et automatiques, 233-250.

Schnedecker, C. et Bianco, M. (1995). Antécédents « dispersés » et référents conjoints ou la construction mentale et la reprise pronominale des entités plurielles. Sémiotiques, 8, 79-107.

Schnedecker, C. (1997). Nom propre et chaînes de référence. Paris : Klincksieck.

Zribi-Hertz, A. (1986). Relations anaphoriques en français : esquisse d'une grammaire générative raisonnée de la réflexivité et de l'ellipse structurale. Doctorat d'Etat Paris 8. Lille : ANRT.

Zribi-Hertz, A. (1996). L'anaphore et les pronoms : une introduction à la syntaxe générative. Villeneuve-d'Ascq (Nord) : Presses universitaires du Septentrion.

\footnotetext{
${ }^{1}$ En français moderne, la cataphore au sens strict pourrait être définie comme la présentation d'un référent sous la forme d'une expression anaphorique sémantiquement incomplète mentionnant le référent comme 'connu' en première mention puis le reprenant ensuite à l'aide d'une expression anaphorique sémantiquement pleine permettant son identification («Lorsqu'elle parlait, Claire tremblait. »). Nous savons que la cataphore - avec l'anaphore - est un phénomène diaphorique pour reprendre le terme de Maillard (1974 et 1987) et ce phénomène n'a pas pu être relevé dans la langue des textes d'AF. Pourtant il semble qu'en MF, d'après nos relevés, ce mode de référenciation voit le jour (Dupuy-Parant, à paraître a). Cependant, les occurrences que nous trouvons en MF ne correspondent pas à la cataphore au sens strict, telle que nous la connaissons en français moderne - définition de Marek Kesik (1989). Cette cataphore du FM n'existait pas en ancien français comme l'a récemment montré B. Combettes (2003) et il semble qu'elle n'existait pas non plus sous cette forme en MF : les occurrences que nous avons pu relever en MF se rapprochent plus de la cataphore au sens large - toujours telle que la définit Marek Kesik (1989) - ou de ce qu'il appelle ana-cataphore. La période du MF semble être une période d'émergence pour certains procédés liés à la continuité référentielle d'où notre intérêt dans le cadre de l'étude des expressions anaphoriques.

${ }^{2}$ Références du corpus : 5 premières joies des $Q J M$ (Droz, 1967), chap. 247 à 261 inclus du livre I des Chr. (Droz, 1972), et 8 premiers chap. du livre III des Mém. (Lettres gothiques, 2001).

${ }^{3}$ Cette comparaison dialectale est moins évidente car le texte des $Q J M$ a vu ses traits dialectaux du Nord Poitou lissés par l'éditeur (Rychner, 1967 : XXXVII) et le texte des Mém. est dans une langue du XV ${ }^{\text {ème }}$ siècle qui ne présente déjà plus de traits dialectaux apparents (éd. Blanchard, 2001 : 62-63 et C Paris, BNF, ms., fr. 5063 - fin XV ${ }^{\text {ème }}$ siècle). Pourtant, par opposition, le texte des Chro., de scripta picarde, se démarque des QJM et des Mém. par certains relevés sur l'usage de catégories de propositions syntaxiques (Dupuy-Parant, 2006 : chap. 6).

${ }^{4}$ Menée à partir du même corpus et vérifiée sur d'autres textes comme le Decameron de Bocasse traduit par L. de Premierfait (1411-1414) (Dupuy-Parant, 2008c).

${ }^{5}$ Dupuy-Parant, 2006.

${ }^{6}$ Expressions anaphoriques réparties en trois catégories :
}

- les anaphores nominales répondant à deux catégories référentielles différentes : redéfinitions (anaphore lexicale infidèle, pronom indéfini, déterminant démonstratif suivi d'un nom renommant, anaphore avec déterminant ledict) - sont incluses les anaphores indirectes ou associatives (Kleiber, 2001) assez rares - et réinitialisations (anaphore lexicale fidèle propre ou impropre sans ledict) - les réinitialisations évitent l'ambiguité référentielle.

- les anaphores pronominales réalisées par le pronom personnel, le pronom relatif et le pronom démonstratif seul.

- les anaphores zéro pour lesquelles seule la désinence verbale (non ambigüe en MF) permet d'identifier la personne verbale.

Pour des raisons de volume et de difficultés de relevé (manuel), nous avons retenu uniquement les expressions anaphoriques induisant des chaînes anaphoriques de personne 3 ou 6 .

${ }^{7}$ Exemple : animé humain...

${ }^{8}$ Ces autres règles prennent en compte le chaînage valentiel des verbes : sont alors à observer les expressions anaphoriques se succédant dans la chaîne d'un même rôle valentiel. Ainsi, un référent qui se maintient en position d'actant 1 d'un verbe à l'autre sera repris par anaphore pronominale ou 'zéro' alors que si un autre référent prend ce rôle valentiel il apparaît sous forme nominale. Il en va de même pour le rôle d'actant 2 indépendamment de celui de l'actant 1 . Ces deux règles s'appliquent d'un verbe à l'autre - sachant qu'un verbe ne peut avoir deux actants de même identité référentielle selon une troisième règle - d'après la catégorie grammaticale de la proposition d'accueil $\mathrm{du}$ verbe. Les propositions non régies, temporelles et relatives en lequel reçoivent pour la distribution valentielle de 
leur verbe l'influence de celle du verbe précédent et influencent celle du verbe les suivant. Les autres propositions régies ne peuvent transmettre l'influence de leur distribution valentielle à celle du verbe suivant.

${ }^{9}$ Parfois, nous observerons également la fusion de référents en un groupe référentiel et son évolution.

${ }^{10}$ Dupuy-Parant, 2006 : QJM 160-164, Chro. 207, Mém. 276.

${ }^{11}$ Ce nombre d'occurrences n'est pas exaustif mais s'approche du nombre réel d'occurrences relevables dans notre corpus. Il correspond uniquement aux groupes référentiels dont l'un des référents constitutifs a été extrait. Ce travail de relevé est très long étant donné qu'il est manuel. Malgré cela, les résultats que nous obtenons présentent une grande cohérence et ouvrent à d'autres tests.

${ }^{12}$ Les relevés dans nos trois textes ne prennent en compte que les référents qui donnent suite à une chaîne anaphorique.

${ }^{13}$ Il semble donc que l'augmentation du nombre de référents extraits (25 dans les Chro. et 46 dans les Mém.) ne soit pas liée à la fréquence des groupes référentiels puisque le nombre des groupes référentiels est très proche dans les Chro. (54 occ.) et les Mém. (52 occ.).

${ }^{14}$ Trois occurrences dans les $Q J M$.

${ }^{15}$ Idem : QJM, p. 16-17, 1. 74-100: «les galans > chacun»; QJM, p. 31, 1. 154-162: «les enfants > ung des petis enfans $>$ celuy que le bon home ayme myeux > le », extrait dans lequel le pronom numéral ung marque l'indéfinitude déterminée par le complément des petis enfans (groupe référentiel d'origine). Une fois présenté comme défini sous la forme celuy que le bon home ayme mieulx, la chaîne anaphorique de ce référent peut se poursuivre sous forme pronominale.

${ }^{16}$ Idem : Chro., p. 835, 1. 1-12: «chil de Calais > li plus poissans et li plus fors > l'un (...) l'autre > li pluiseur» formation de sous-groupes référentiels ne représentant pas la totalité du groupe matrice mais pouvant présenter des intersections d'ensembles les uns entre les autres. Chro., p. 842-843, 1. 16-28: «ils tous > honmes et fenmes > il (...) tout $>$ honmes et fenmes $>$ il $>$ tout et toutes $\gg$ où le groupe matrice est scindé en deux sous-groupes complémentaires appuyés par l'opposition de genre.

${ }^{17}$ Idem dans les Mém., p. 244, 1. 15-19: «Ledict de Vaucloe me manda a disner, qui estoit bien acompaigné, et avoit le revastre d'or sur son bonnet (qui estoit la livree dudict conte), qui est ung baston noir, et tous les aultres semblablement; et qui ne le pouvoit avoir d'or, l'avoit de drap. » où le groupe référentiel matrice catégorisant dont les entités référentielles sont indéfinies, est scindé en deux sous-groupes référentiels complémentaires et dont l'addition correspond à la totalité du groupe matrice : tous les aultres semblablement $(\mathrm{Gp} 0)=$ qui ne le pouvoit avoir d'or (Gp1 explicite) + qui [Ø] le pouvoit [l']avoi[t] d'or (Gp2 implicite, opposé complémentaire de Gp1). Le référent 'Ledict de Vaucloe' car [...] avoit le revastre d'or sur son bonnet appartient à Gp2.

${ }^{18}$ Mém., p. 256, 1.29 - p. 257, 1. 6: «l'armee > douze cens lances d'ordonnance > trois archiers pour homme d'armes > le tout / Chascune compaignee > dix hommes d'armes > le lieutenant et ceulx qui portoient les enseignes »

${ }^{19}$ Chro., p. 850-851, 1. 20-28: «chil chevalier françois > mesire Jehans de Viane et tout li chevalier de France » si nous admettons que le groupe référentiel «chil chevalier françois» correspond au sous-groupe référentiel «tout li chevalier de France ».

${ }^{20}$ Voir la règle de concurrence référentielle définie en préambule.

${ }^{21}$ Références des groupes référentiels de forme «ils et + Substantif» dans les Chro.: p. 823, 1. 109: ils et ses consauls; p. 824, 1.9: ils et ses gens; p. 833, 1. 48 : ils ne ses gens; p. 845, 1. 102-103: ils et li siis bourgeois.

22 «Pendant toute la période du moyen français, ce tour [« moy et ce petit enfant», Griseldis, v. 1374)] coexiste avec le tour ancien : je/tu/...+ et $+X$ : Froissart (ou son copiste) écrit aussi bien il et ses gens que luy et sa route [...] ce n'est qu'au XVI ${ }^{\mathrm{e}}$ siècle en effet que cette évolution s'achève avec la disparition des pronoms de forme I dans ce tour, je étant le dernier à y être employé. » (Marchello-Nizia, $1997: 243$ ).

${ }^{23}$ Deux autres exemples dans le livre III des Mém. pour lesquels le référent identifié correspond à l'auteur/narrateur et apparaît donc sous la forme pronominale d'un pronom personnel de personne 1 et est extrait par anaphore pronominale : p. 243, 1. 5-9: Ledict duc me renvoya incontinent a Callais, et ung gentilhomme ou deux qui estoient de ceste parcialité nouvelle de Henry et me commenda ce qu'il vouloit que je feisse avecques ce monde neuf; p. 244, 1. 2-6: le cappitaine [...] feist tres grand chere et honneur a ces gentilz hommes qui estoient avecques moy des partisans du roy Henry. Je allay a Callays.

${ }^{24}$ QJM, p. 33, 1. 15-27: "elle + le mari > ilz > elle lui dit»; QJM, p. 35, 1. 75-89: «ilz> ensemble > il »; QJM, p. 38-39, 1. 198-208: «ilz > la dame > il > elle»; QJM, p. 44-45, 1. 293-408: «parlent ensemble > elle»; QJM, p. 35-36, 1. 97-102.

${ }^{25}$ Chro., p. 812, 1. 87-90.

${ }^{26}$ Est constitué un groupe référentiel matrice messires Thomas d'Angourne, messires Jehans de Hartecelle et messires Tangis dou Chastiel et tout li chevalier et esquier de lor route, qui la estoient assamblé redéfini ensuite sous la forme nominale ces Englois et Bretons (concurrence référentielle avec le groupe 'des français'). Et de ce groupe 
978-2-7598-0534-1, Paris, 2010, Institut de Linguistique Française

Diachronie

DOI $10.1051 / \mathrm{cmlf} / 2010167$

matrice est extrait de façon intermédiaire un sous-groupe référentiel sous forme pronominale euls immédiatement repris par anaphore nominale, li troi chevalier, identification confirmée par l'anaphore nominale suivante des trois chevaliers desus nonmés, qui réfère aux entités précédemment mentionnées, messires Thomas d'Angourne, messires Jehans de Hartecelle et messires Tangis dou Chastiel.

${ }^{27}$ Kesik, 1989.

${ }^{28}$ La fusion est réalisée également à travers cette expression anaphorique par le contexte situationnel qui fait des deux référents 'roi' et 'duc de Bourgogne' des référents contextuellement opposés au référent 'connestable'.

${ }^{29}$ Dupuy-Parant, 2008b.

${ }^{30} C f$. note 29 